Published in final edited form as:

Yan, R., Antonietti, M., \& Oschatz, M. (2018). Toward the Experimental Understanding of the Energy Storage Mechanism and Ion Dynamics in Ionic Liquid Based Supercapacitors.

Advanced Energy Materials, 8(18): 1800026. doi:10.1002/aenm.201800026.

\title{
Toward the Experimental Understanding of the Energy Storage Mechanism and Ion Dynamics in Ionic Liquid Based Supercapacitors
}

Runyu Yan, Markus Antonietti, Martin Oschatz

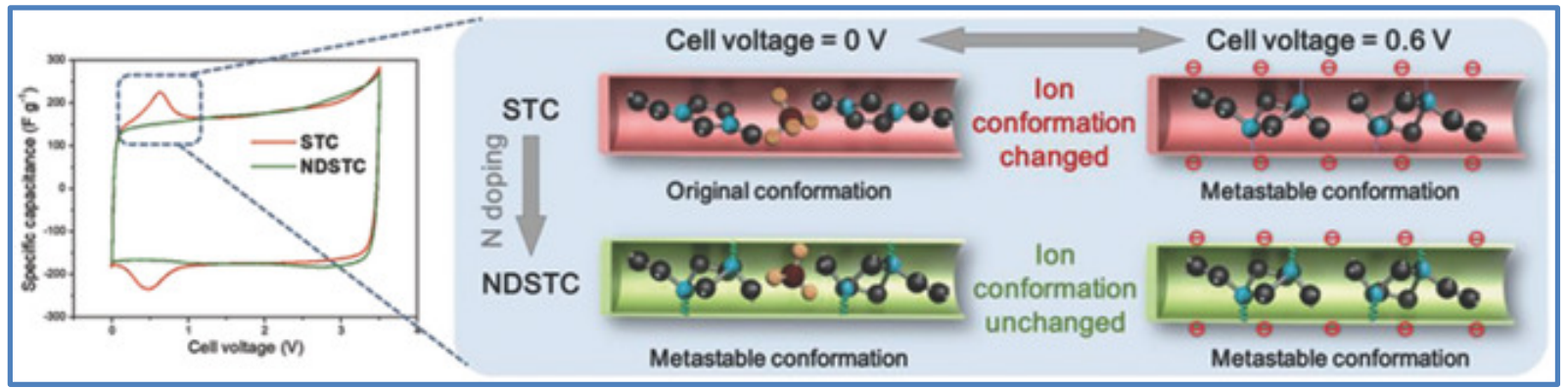

In electric double-layer capacitors with ionic liquid electrolytes, ion conformation change and phase variation during charging/discharging are uncovered, which significantly strengthen charge storage in the electric double-layer and supplement the traditional energy storage mechanism. A quantitative model of the structuredynamics relationship is proposed, in which the optimal ratio of mesopores to micropores is determined to be $3: 1$ in pore volume.

This article may be used for non-commercial purposes in accordance with Wiley Terms and Conditions for Self-Archiving. 


\title{
Towards the experimental understanding of the energy storage mechanism and ion dynamics in ionic liquid based supercapacitors
}

\author{
Runyu Yan, Markus Antonietti, Martin Oschatz*
}

\author{
R. Yan, Prof. Dr. Dr. M. Antonietti, Dr. M. Oschatz \\ Max Planck Institute of Colloids and Interfaces \\ Department of Colloid Chemistry \\ Am Mühlenberg 1, 14476 Potsdam \\ Germany \\ E-mail: martin.oschatz@mpikg.mpg.de
}

Keywords: electric double-layer capacitors, nitrogen-doped porous carbon, salt-templated approach, ion storage/transport mechanism, ion conformation variation

A series of salt-templated carbons with gradually changed pore structure and their corresponding nitrogen-doped analogues are synthesized and applied as model systems to thoroughly study the ion migration dynamics and energy storage mechanism in hierarchical pore structures with different surface functionalization in electric double-layer capacitors with a model ionic liquid electrolyte (1-ethyl-3-methylimidazolium tetrafluoroborate).

Ion conformation and phase variation during the charging/discharging process and their contribution to the energy storage mechanism are investigated. A significant contribution of structural changes in the bulk of the ionic liquid electrolyte strengthening charge storage in the electric double-layer beyond the usual expectations is uncovered.

Furthermore, a quantitative model of the structure-dynamics relationship is proposed, in which the optimal ratio of mesopores to micropores is determined to be $3: 1$ in pore volume. Below this ratio, the ion dynamics can be promoted by increasing mesopore content and/or doping with nitrogen, while those parameters show only minor influence when the ratio is surpassing 3:1. Nitrogen doping in this system improves the rate capability (due to the enhanced ion transport dynamics) rather than the amount of energy stored. 


\section{Introduction}

Owing to the increasing electrification of the world, there is an ever-growing demand for high-performance, cost-effective, and environmentally-friendly electrochemical energy storage systems. Electric double-layer capacitors (EDLCs), which store charges at the electrode/electrolyte interface through reversible ion adsorption on porous conductive electrodes, are energy storage devices offering exceptionally large power densities that can be used to harvest energy produced by intermittent renewable energy sources and for energy recovery on short time scales. ${ }^{[1]}$ Porous carbons have been the prime choice of the electrode materials for EDLCs owing to their versatile porosity, high surface area, and good electronic conductivity. ${ }^{[2]}$ However, most often the inferior energy density of EDLCs poses restrictions for some of their possible large-scale applications. Given the square dependence of the energy density on applied voltage windows for EDLCs, a key strategy to enhance energy storage capacity is to implement electrolytes with higher electrochemical windows and stabilities. Ionic liquids (ILs), also known as low temperature molten salts (melting points below $100{ }^{\circ} \mathrm{C}$ ) are composed solely of ions and thus display promising alternatives to the established solventbased systems due to their wide operation voltage (as high as $4 \mathrm{~V}$ ), negligible volatility, nonflammability, as well as the large potential operating temperature range. ${ }^{[3]}$

An issue of such electrolytes, however, is that they tend to lower the power density of EDLCs. Typically, ion transport in ILs is slow in the bulk and could be even slower in carbon nanopores, leading to sluggish charging dynamics and lowered power density. Resolving these issues necessitates the fundamental understanding of transport dynamics in nanopores filled with ILs. For example, while most studies demonstrate better IL dynamics in larger pores, ${ }^{[4]}$ molecular simulation disclosed ultrahigh transport coefficient in charged micropores as well. ${ }^{[5]}$ The ion dynamics also depend on the surface chemistry where both the ionophobic carbon micropores and hydrophobic silica mesopores provide surprisingly high migration 
coefficients of IL ions. ${ }^{[6]}$ Molecular dynamics simulation has revealed the dynamic heterogeneity of IL confined inside uncharged mesopores where the dynamics slow down appreciably as the ions approach the pore walls, ${ }^{[7]}$ as well as the great variation of the ion occupancy (total ion density) inside pores with different charging rates. ${ }^{[8]}$ These works attracted much attention and have contributed significantly to the progress toward the elucidation of how the size, surface chemistry, charging state, ion-packing density, ${ }^{[9]}$ and other physicochemical properties of the pores, affect the charge storage mechanism and charge transport dynamics in IL-filled supercapacitors. However, the nanopores in all these theoretic studies are monodisperse in pore size without hierarchy, while hierarchical pore structure with both micro- and mesopores have been proven experimentally to provide efficient ion transport for a high power density while maintaining the high energy density. ${ }^{[10]}$ There is still a lack of systematic studies and comprehensive interpretation of the dynamics of electrolyte ions in such complex electrode structures during the charging/discharging process. Understanding requires a combination of computational simulation and experiments, whereas the inadequate characterization of the intricate structure of hierarchical porous carbons without long-range order poses a significant challenge to the modelling and thus the simulation. ${ }^{[11]}$ On the other side, the great difficulty in fabricating nearly ideal carbon models with well-defined hierarchical porosity as well as precisely controlling their pore structures impedes an experimental probing of the sophisticated hierarchical pore structure-property relationships.

From the experimental and material perspective, heteroatom (especially nitrogen) doping of the carbon atom framework has been acknowledged as another efficient approach to improve both the capacitance and rate capability of supercapacitors. ${ }^{[12]}$ Nevertheless, all the above fundamental research on charge storage mechanisms has been devoted exclusively to pure carbon materials. A deep comprehension of a potential change of mechanisms after nitrogen doping remains missing. 
Numerous pioneering experimental and theoretical studies have been devoted to explore the underlying physics for IL charge storage under equilibrium conditions in carbon nanopores, which have led to an improved understanding of the molecular mechanisms of charge storage. For instance, powerful techniques such as computer simulations, ${ }^{[13]}$ electrochemical quartz crystal microbalance, ${ }^{[14]}$ nuclear magnetic resonance (NMR) spectroscopy, ${ }^{[15]}$ and infrared spectroelectrochemical techniques ${ }^{[16]}$ have provided molecular information about the dynamic charge storage, where the ion exchange (swapping of co-ions for counter-ions) seems to play a vital role. ${ }^{[1 \mathrm{~d}, 17]}$ Great efforts have been devoted to investigating the dependence of capacitance on the pore size for an ionic liquid electrolyte, especially the anomalous increase of capacitance when the pore size decreases to the ion size. ${ }^{[18]}$ Using molecular dynamics simulations, the capacitance is found to depend on the applied voltage as well, where the capacitance reaches a maximum when all co-ions are expelled from the pores. ${ }^{[13]}$ These reports emphasize the significance of the charging process, applied voltage, pore sizes, ionwall/ion-ion interactions, and physico-chemical properties of the IL, just to name a few. Up to now, the mechanisms for charge storage revealed in these studies neglect possible conformation variations and phase changes in the bulk of the rather large IL ions during charging/discharging. Simulation studies only employ coarse-grained, rigid models of IL ions, and experimental studies have not taken the influence of such variations into consideration, although they are very likely to occur as a function of the strength of ion-ion and/or ion-wall interactions in presence of an electric potential. A limited number of simulation studies have revealed that the ion-wall interactions can significantly affect the molecular conformation of IL ions. ${ }^{[5,7 b]}$ Nevertheless, no experimental results confirming this prediction have been reported, and its importance for the energy storage mechanism as well as the origin remains ambiguous.

In this work, a series of salt-templated carbon materials (STCs) and their corresponding nitrogen-doped counterparts (NDSTCs) with tunable pore structure and well-defined pore size 
dispersion were synthesized and used as model systems to thoroughly study the dynamics of the ion transport in hierarchical pore structures with different surface functionalization. The ion conformation and possible phase variations in the bulk of the IL confined in the carbon pores during charging/discharging process and its contribution to the charge storage mechanism was investigated as well. A quantitative model is proposed for the relationship between hierarchical pore structure/surface functionalization and ion dynamics. The increasing amount of mesopores and doping with nitrogen can promote the amount of energy stored in the EDLC only when it is limited by the inferior ion dynamics inside micropores. The ion migration dynamics inside mesopores commence to limit the EDLC performance when the ratio of mesopores to micropores surpasses a critical point of 3:1 in volume. Above this ratio neither increase of mesopore volume nor nitrogen doping will have significant influence. In terms of the energy storage itself, the occurrence of ion conformation variation during charging/discharging process leads to an unexpected deviation of the energy storage mechanism from the classical double-layer theory due to significant contributions from the intermolecular interactions in the IL volume.

\section{Results}

A series of salt-templated carbons $(\mathrm{STCs})^{[19]}$ with different pore structures was prepared by employing the salt template $\mathrm{ZnCl}_{2}$ as porogen for well-defined micropores and mesopores (Scheme 1). Sucrose as a renewable carbon precursor and different amounts of $\mathrm{ZnCl}_{2}$ were mixed in aqueous solution, followed by polycondensation of the carbohydrate at $160{ }^{\circ} \mathrm{C}$ with the salt template inside. Afterwards, the mixture was carbonized at $900{ }^{\circ} \mathrm{C}$ followed by thorough washing with $\mathrm{HCl}$ solution to remove the $\mathrm{ZnCl}_{2}$. Thermogravimetric analysis (TGA) of the materials indicated a small ash-content (less than $3 \mathrm{wt} \%$ ) above $600{ }^{\circ} \mathrm{C}$ (Figure S1). Xray diffraction (XRD) results (Figure S2) of all the samples showed only broad (002) and (101) carbon peaks at $26^{\circ}$ and $44^{\circ} 2$ Theta, verifying the mainly amorphous carbon phase and 
the nearly complete absence of inorganic residuals after carbonization and washing with $\mathrm{HCl}$. Removal of the salt leads to the formation of micropores and mesopores at controllable ratio depending on the relative amounts of $\mathrm{ZnCl}_{2}$ in different STCs (Table 1). This synthesis scheme stands out due to the tunable hierarchical pore structure formation with high surface area by a simple one-step salt-template approach using a potentially reusable template $\mathrm{ZnCl}_{2}$. Reasonable yields of carbon can be achieved from a renewable precursor, which is sustainable, cost-effective, and favorable to mass production. Subsequently, nitrogen doping of STCs was carried out by dispersing the STCs in aqueous cyanamide solution followed by drying and cross-condensation at $800{ }^{\circ} \mathrm{C}$ under $\mathrm{N}_{2}$ to obtain the corresponding nitrogen-doped STCs (NDSTCs, Scheme 1). The nitrogen content is $5-6 \mathrm{wt} \%$ for all these samples, as detected by elemental analysis (Table 1).

X-ray photoelectron spectroscopy (XPS) was employed to characterize the N-doped samples (NDSTC-1 and NDSTC-16) for the nitrogen/oxygen containing functional groups. The survey XPS spectra (Figure S3a and d) of the two samples reveal the presence of C, N, and O. Traces of residual zinc are also detected which is consistent with the results of TGA. The high-resolution N 1s spectra (Figure S3b and e) of both NDSTCs display four fitted peaks at 397.9, 398.5, 400.1, and $403.2 \mathrm{eV}$, which can be assigned to the pyridinic nitrogen, pyrrolic nitrogen, graphitic nitrogen, and N-O (oxidized nitrogen), respectively. ${ }^{[20]}$ The high-resolution O 1s spectra (Figure S3c and f) can be deconvoluted into two peaks centered at 531.5, and $532.8 \mathrm{eV}$, corresponding to the carbonyl groups $(\mathrm{C}=\mathrm{O})$ and hydroxyl groups $(\mathrm{C}-\mathrm{OH})$, respectively. ${ }^{[21]}$ The nitrogen contents of NDSTC-1 and NDSTC-16 are determined to be 6.01 and 3.68 at $\%$, respectively, which suggests the success of nitrogen doping since negligible amount of nitrogen was detected in STC-1 and STC-16 by XPS in accordance with the results of elemental analysis.

$\mathrm{N}_{2}\left(-196{ }^{\circ} \mathrm{C}\right)$ and $\mathrm{CO}_{2}\left({ }^{\circ} \mathrm{C}\right)$ physisorption experiments were carried out to analyze the pore structures (Figure 1, Figure S4 and Table 1). STC-1 shows type I(a) isotherm, indicating its 


\begin{tabular}{|c|c|c|c|c|c|c|c|c|}
\hline & \multirow{2}{*}{$\begin{array}{l}\mathrm{ZnCl}_{2} \text { :Sucrose } \\
\text { (weight) }\end{array}$} & \multirow{2}{*}{$\begin{array}{c}\text { SSA } \\
\left(\mathrm{m}^{2} / \mathrm{g}\right)\end{array}$} & \multirow{2}{*}{$V_{t}\left(\mathrm{~cm}^{3} / g\right)$} & \multicolumn{2}{|c|}{ DFT } & \multirow{2}{*}{$\begin{array}{c}\text { N content } \\
(\mathbf{w t} \%)\end{array}$} & \multirow{2}{*}{$\begin{array}{c}\text { Thickness } \\
(\mu \mathrm{m})\end{array}$} & \multirow{2}{*}{$\begin{array}{l}\text { Mass loading } \\
\left(\mathrm{mg} \mathrm{cm}^{-2}\right)\end{array}$} \\
\hline & & & & $V_{m i c}\left(\mathrm{~cm}^{3} / \mathrm{g}\right)$ & $V_{\text {meso }}\left(\mathrm{cm}^{3} / \mathrm{g}\right)$ & & & \\
\hline STC-1 & 1 & 1138 & 0.51 & 0.39 & 0.11 & / & 126 & 9.6 \\
\hline STC-2 & 2 & 1430 & 0.81 & 0.33 & 0.42 & l & 122 & 7.6 \\
\hline STC-4 & 4 & 1739 & 1.08 & 0.36 & 0.67 & l & 125 & 5.7 \\
\hline STC-8 & 8 & 2203 & 1.48 & 0.36 & 1.08 & / & 118 & 5.0 \\
\hline STC-16 & 16 & 2324 & 1.66 & 0.41 & 1.25 & 1 & 123 & 4.8 \\
\hline NDSTC-1 & 1 & 1163 & 0.51 & 0.39 & 0.08 & 5.40 & 130 & 11.6 \\
\hline NDSTC-2 & 2 & 1468 & 0.80 & 0.37 & 0.38 & 5.52 & 123 & 6.9 \\
\hline NDSTC-4 & 4 & 1811 & 1.09 & 0.42 & 0.62 & 5.74 & 126 & 6.2 \\
\hline NDSTC-8 & 8 & 2181 & 1.42 & 0.36 & 1.02 & 6.09 & 119 & 5.0 \\
\hline NDSTC-16 & 16 & 2308 & 1.63 & 0.40 & 1.15 & 5.43 & 121 & 4.8 \\
\hline Ac-STC-1 & 1 & 1493 & 0.72 & 0.50 & 0.18 & / & 122 & 8.6 \\
\hline Ac-STC-16 & 16 & 2833 & 1.89 & 0.48 & 1.35 & 1 & 116 & 3.2 \\
\hline
\end{tabular}

Table 1. Mass ratio of $\mathrm{ZnCl}_{2}$ to sucrose, BET Specific surface area (SSA), total pore volume $\left(\mathrm{V}_{\mathrm{t}}\right)$, DFT micropore volume $\left(\mathrm{V}_{\text {mic }}\right)$, DFT mesopore volume $\left(\mathrm{V}_{\text {meso }}\right)$, nitrogen content of the carbon materials, as well as the thickness and mass loading of the corresponding electrodes.

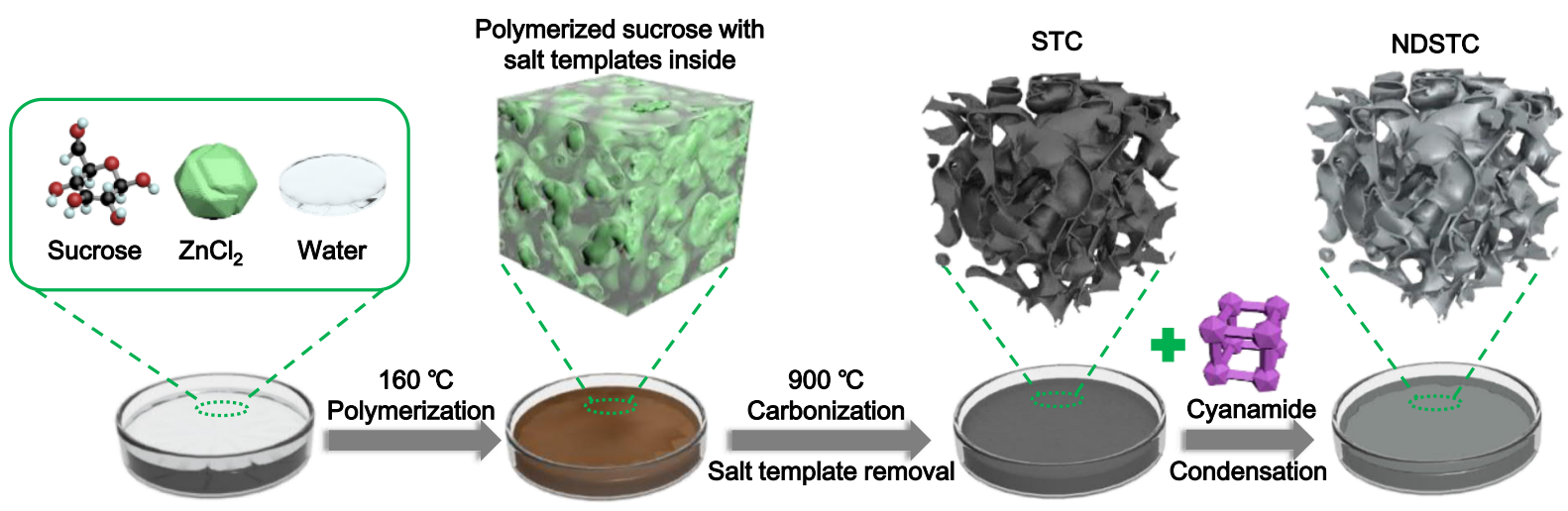

Scheme 1. Preparation of salt-templated carbons (STCs) and nitrogen-doped STCs (NDSTCs). 
purely microporous structure, while STC-2 has the type I(b) isotherm which suggests the presence of a small amount of narrow mesopores in addition to the micropores. STC-4, STC- 8 and STC-16 exhibit type IV(a) isotherms with a continuous slope above a relative pressure of 0.2 with the hysteresis loop of $\mathrm{H} 4$ type in the relative pressure range between 0.4 and 0.9 , indicating the existence of significant amounts of mesopores (Figure 1a). To study the transformation of pore structure with the increase of the salt template, the pore size distribution (PSD) was further analyzed using the quenched-solid density functional theory (QSDFT) for $\mathrm{N}_{2}$ adsorbed on carbon with slit/cylindrical pore shape (Figure 1b). All the STC samples exhibit comparable micropore volumes around $0.4 \mathrm{~cm}^{3} \mathrm{~g}^{-1}$ (Table 1). In the case of STC-1 with the moderate SSA of $1138 \mathrm{~m}^{2} \mathrm{~g}^{-1}$, the micropores account for the overwhelming fraction of the total pores (Figure 1c), verifying its microporous pore structure. With the increase of the salt loading, the specific surface areas (SSAs) gradually and significantly increase to $2203 \mathrm{~m}^{2} \mathrm{~g}^{-1}$ for STC-8, followed by $2324 \mathrm{~m}^{2} \mathrm{~g}^{-1}$ for STC-16 (Figure 1g and Table 1). The content of mesopores exhibits a drastic rise with increased template content (Figure 1h), which in turn results in a continuous augment of the total pore volumes from $0.51 \mathrm{~cm}^{3} \mathrm{~g}^{-1}$ for STC-1 to $1.66 \mathrm{~cm}^{3} \mathrm{~g}^{-1}$ for STC-16. In addition, the diameters of the mesopores are also enlarged slightly from $3 \mathrm{~nm}$ (STC-2) to $4 \mathrm{~nm}$ (STC-16) (Figure 1b) which is likely due to percolation of the salt templates, allowing to fine tune the hierarchical pore structure. The cumulative PSD plots (Figure 1c) indicate that micropores only contribute a small fraction of the total pore volume in the STC-4, STC-8, and STC-16. The distributions of micropores with diameters of $1.5 \mathrm{~nm}$ and below were further evaluated by non-local DFT (NLDFT) analysis of $\mathrm{CO}_{2}$ physisorption measurements (Figure 1d-f). All samples contain three kinds of welldefined narrow micropores with diameters of $0.35,0.55$, and $0.80 \mathrm{~nm}$, corresponding to different well-defined salt template clusters (Figure 1e). A higher $\mathrm{ZnCl}_{2} /$ sucrose ratio leads to a larger volume of micropores with the diameter of $0.80 \mathrm{~nm}$, which is in the range of the ion size of $\mathrm{EMIm}^{+}(0.76 \mathrm{~nm})$. This underlines that the salt-templating method provides a 
sufficiently precise control over the size and amount of narrow micropores. The $\mathrm{N}_{2}$ physisorption results of NDSTCs show the similarity of pore structure compared with their corresponding parental STCs (Figure S4 and Table 1), indicating that the nitrogen doping process is a molecular cross-condensation which has no significant effects on the pore structure. The presence of comparable amounts of nitrogen in purely microporous NDSTC-1 and other hierarchical porous NDSTCs (Table 1) suggest the homogenous nitrogen doping along both micropores and mesopores.

The influence of the amount of $\mathrm{ZnCl}_{2}$ and nitrogen doping on the pore structure was further studied by transmission electron microscopy (TEM). Low content of $\mathrm{ZnCl}_{2}$ (STC-1 and STC2) leads to a rather dense morphology with the presence of abundant narrow micropores, while mesopores can hardly be observed (Figure 2a-b). With the gradual increase of $\mathrm{ZnCl}_{2}$ (STC-4, STC-8, and STC-16), the morphologies clearly exhibit a distinct trend of being more diluted and open, reflecting the development of open mesoporous pore systems (Figure 2c-e). After nitrogen doping, the NDSTCs consist of pore structures which are comparable to their corresponding STC parents (Figure $\mathbf{2 f - j}$ ). This is in good accordance with the physisorption experiments.

In the Raman spectra (Figure S5), the D-band near $1340 \mathrm{~cm}^{-1}$ originates from the breathing modes of the six-fold $\mathrm{sp}^{2}$-hybridized carbon rings in the presence of defects and disorder. ${ }^{[22]}$ The width of the D-band reflects the degree of structural ordering and size distribution of $\mathrm{sp}^{2}$ rings (i.e., 5-, 6-, 7-, and 8-membered rings). The wide $\left(157-196 \mathrm{~cm}^{-1}\right)$ D-bands (Table S1) in all samples reveal the existence of abundant disordered sections as it is typical for highly microporous carbons. ${ }^{[22 \mathrm{a}]}$ The G-band near $1580 \mathrm{~cm}^{-1}$ is caused by bond stretching of $\mathrm{sp}^{2}$ carbon in either rings or chains. The peak height ratio of the $\mathrm{D}$ - and G-band $\left(\mathrm{I}_{\mathrm{D}} / \mathrm{I}_{\mathrm{G}}\right)$ is proportional to the amount of six-membered $\mathrm{sp}^{2}$ carbon rings, which is commonly employed to evaluate the level of carbon ordering in porous carbons. ${ }^{[22-23]}$ The comparable $\mathrm{I}_{\mathrm{D}} / \mathrm{I}_{\mathrm{G}}$ value (Table S1) with the increase of salt-template (the values of STC-1, STC-2, STC-4, STC-8, 

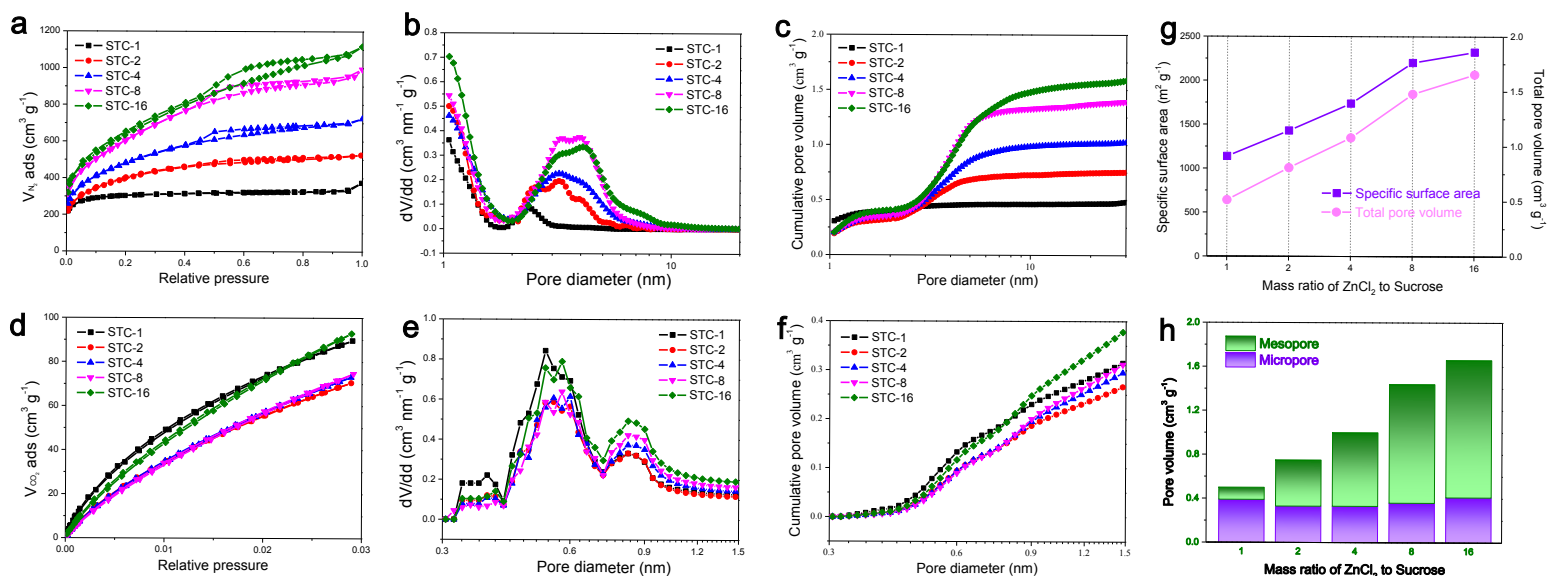

Figure 1. (a) $\mathrm{N}_{2}$ physisorption isotherms (at $-196^{\circ} \mathrm{C}$ ) with corresponding (b) differential and (c) cumulative QSDFT PSDs as well as (d) $\mathrm{CO}_{2}$ physisorption (at $0{ }^{\circ} \mathrm{C}$ ) isotherms with corresponding (e) differential and (f) cumulative NLDFT PSDs of the STCs (h) Micro-/mesopore ratios in the samples.
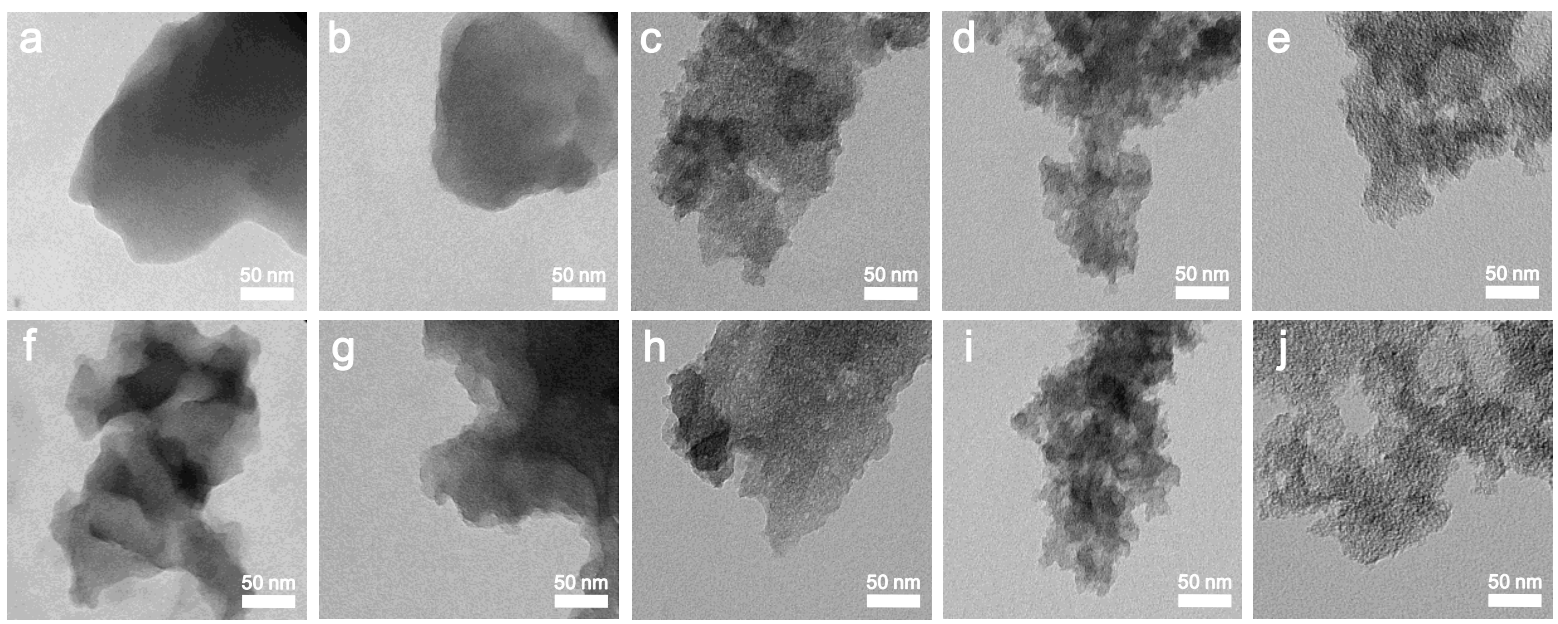

Figure 2. TEM images of the STCs and the respective NDSTCs. (a) STC-1, (b) STC-2, (c) STC-4, (d) STC-8, (e) STC-16, (f) NDSTC-1, (g) NDSTC-2, (h) NDSTC-4, (i) NDSTC-8, (j) NDSTC-16. 
and STC-16 equal 1.3, 1.3, 1.2, 1.1, and 1.3, respectively) indicates comparable degree of aromatization and thereby the similar electric conductivity for these STCs.

To establish structure-performance relationships of the STCs for EDLCs, the carbon samples were fabricated into self-standing electrodes and characterized with cyclic voltammetry (CV) tests, galvanostatic charging/discharging with potential limitation (GCPL), and electrochemical impedance spectroscopy (EIS) in solvent-free ionic liquid (EMImBF 4$)$. All the Nyquist plots are nearly vertical in the low-frequency region except for STC-1 due to its partially inaccessible micropores (Figure S6b). The CV curves show nearly rectangular shapes from 0-3.5 V at different scan rates (Figure S7a-e) and GCPL curves are close to symmetric triangle at a low specific current of $0.2 \mathrm{~A} \mathrm{~g}^{-1}$ (Figure S6a) - a typical characteristic of double-layer capacitor behavior. However, all these CV curves exhibit small peaks at a cell voltage of $\sim 0.6 \mathrm{~V}$ (Figure 3a), which will be attributed to the conformation change of ions inside the micropores during charging/discharging and will be further discussed in the following section. Even more pronounced peaks start to emerge at higher cell voltages. At a low specific current of $0.2 \mathrm{~A} \mathrm{~g}^{-1}$, the specific capacitance of microporous STC-1 reaches 49 $\mathrm{F} \mathrm{g}^{-1}$ as determined by the GCPL measurements. With the increase of the $\mathrm{ZnCl}_{2}$, the specific capacitance shows a drastic rise to $149 \mathrm{~F} \mathrm{~g}^{-1}$ (STC-2) which then levels off to $178 \mathrm{~F} \mathrm{~g}^{-1}$ (STC16), in spite of the further significant increase of the SSAs (Figure 1g). This results in an increase of the specific capacitance normalized by BET surface area from $4.3 \mu \mathrm{F} \mathrm{cm} \mathrm{cm}^{-2}$ (STC1) to $10.4 \mu \mathrm{F} \mathrm{cm} \mathrm{cm}^{-2}$ (STC-2), followed by an apparent drop to $8.9 \mu \mathrm{F} \mathrm{cm}^{-2}$ (STC-4) and $\sim 7.5 \mu \mathrm{F}$ $\mathrm{cm}^{-2}$ (STC-8 and STC-16, Figure 3c). The rather low normalized specific capacitance at 0.2 $\mathrm{A} \mathrm{g}^{-1}$ of STC-1 is ascribed to the restricted accessibility of the electrode surface within the purely microporous structure, with a large portion of the carbon surface being not accessible even during a slow, quasi-equilibrium charging/discharging process. The inferior pore accessibility of STC-1 is also revealed by the clear deviation of the Nyquist plot in the low frequency range (Figure S6b). ${ }^{[24]}$ The drastic rise of normalized specific capacitance from 
STC-1 to STC-2 is caused by the introduction of mesopores, which renders the whole carbon surface available to ions at a low charging/discharging rate. Due to its enlarged SSA and mesopore volume, STC-2 reaches a relatively high specific capacitance of $149 \mathrm{~F} \mathrm{~g}^{-1}$. It is worth mentioning that further increase of the SSA only gives rise to the slight improvement of specific capacitance, leading to the decline of normalized specific capacitance to approximate $7.5 \mu \mathrm{F} \mathrm{cm}{ }^{-2}$ for STC-8 and STC-16 (Figure 3c). This is consistent with the rather general observation that an "endless" increase of SSAs above $1500 \mathrm{~m}^{2} \mathrm{~g}^{-1}$ has only small impacts on the specific capacitance, and the pore size distribution becomes crucial. ${ }^{[1 \mathrm{a}, 1 \mathrm{e}]}$ This is also supported by analysis of a $\mathrm{CO}_{2}$-activated STC-16 (Ac-STC-16) which "only" reaches $154 \mathrm{~F} \mathrm{~g}^{\text {- }}$ ${ }^{1}$ (Figure 4) despite its ultrahigh SSA of $2833 \mathrm{~m}^{2} \mathrm{~g}^{-1}$. On base of the normalized specific capacitance of $5.4 \mu \mathrm{F} \mathrm{cm}$, this material provides an even lower specific capacitance compared with that of STC-16 (Figure 4b and Figure 4c). ${ }^{[18 e]}$

The rate capabilities from STC-1 to STC-8 show a clear improvement with the higher amounts of the mesopores, which is seen by the smaller voltage drop (Figure S6a) and higher capacitance retention (Figure 3b-c). Typically, in the case of STC-1, STC-2, and STC-4, only part of the surface in the micropores is used by ions at a high charging/discharging rate because of the poor ion dynamics. This can be addressed by the substantial development of mesopores (introduced by increasing amounts of salt template), leading to the shrinkage of domains of micropores, thus significantly improving the rate capability. It should be noticed that the larger areal loading (at similar thickness) of the electrodes with the microporous carbons could also contribute their worse rate capability by decreasing migration dynamics of charge carriers, especially at very high current density. On the other hand, STC-8 and STC-16 exhibit similar rate capabilities with polarization induced voltage drops of $0.34 \mathrm{~V}$ and $0.37 \mathrm{~V}$, and capacitance retentions of $87 \%$ and $89 \%$, respectively, at a specific current of $5 \mathrm{~A} \mathrm{~g} \mathrm{~g}^{-1}$, despite their apparent difference in content of mesopores (Figure 1h). This is in accordance with the Nyquist plots of the STCs (Figure S6b), where the length of the $45^{\circ}$ sloped curve 

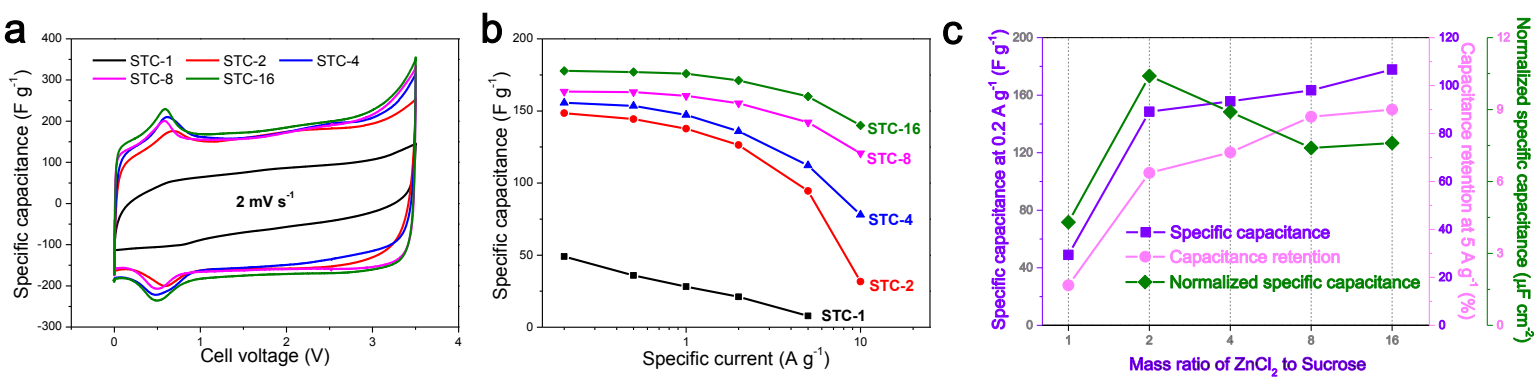

Figure 3. EDLC performance of STCs tested in $\mathrm{EMImBF}_{4}$ ionic liquid using a two-electrode configuration: (a) Cyclic voltammograms at $2 \mathrm{mV} \mathrm{s}^{-1}$, (b) capacitance retention with specific current increase, and (c) influence of the amount of $\mathrm{ZnCl}_{2}$ on the specific capacitance, capacitance retention, and specific capacitance normalized by the BET SSAs of the obtained STCs.
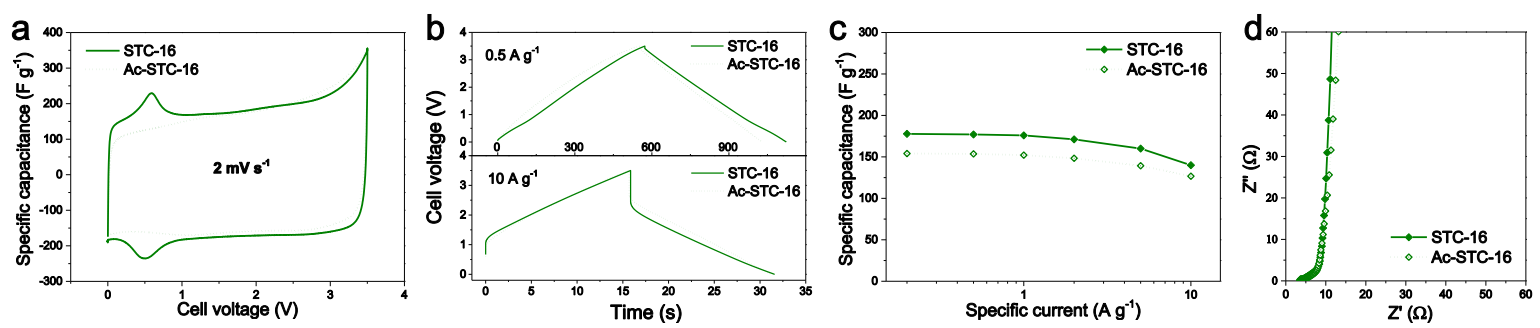

Figure 4. EDLC performance comparison of STC-16 and Ac-STC-16 tested in EMImBF 4 ionic liquid using a two-electrode configuration: (a) Cyclic voltammograms at different scan rates, (b) charge/discharge profiles at different specific currents, (c) capacitance retention with specific current increase, and (d) Nyquist plots. 
(known as the Warburg portion in the middle-frequency region, related to the transport of electrolyte ions into the carbon pores) shows an apparent reduction from $10.9 \Omega$ for STC-1 to $4.9 \Omega$ for STC-8, while constancy of this value $(\sim 4.9 \Omega)$ is observed for STC-8 and STC-16. This suggests that the further shortening of transport length in the micropores in STC-8 has little influence on the rate capability. To identify the rate determining process, we tested the EDLC performance with Ac-STC-16 as an "activated" electrode material where the micropore sizes are slightly enlarged with broader distribution (Figure S8), so that the ion transport dynamics at the junctions of mesopores and micropores are expected to be improved. However, the rate capability is almost impervious to the enlargement of micropores, as revealed by the similar voltage drop $(1.1 \mathrm{~V}$ and $1.0 \mathrm{~V})$, and capacitance retention $(79 \%$ and 81\%) of STC-16 and Ac-STC-16 at $10 \mathrm{~A} \mathrm{~g}^{-1}$, as well as their similar Nyquist plots (Figure 4b-d). The slightly lower capacitance retention of STC-16 is attributed to the absent peak at the high charging/discharging rate (Figure S7e). Besides, in our recent study, a hierarchical porous carbon with ordered mesopores shows outstanding rate capability $(0.64 \mathrm{~V}$ voltage drop and $88 \%$ capacitance retention at $10 \mathrm{~A} \mathrm{~g}^{-1}$ ), compared with that of the STCs with disordered and relatively narrow mesopores. ${ }^{[10 \mathrm{c}]}$ Therefore, we conclude that the limiting factor of the ion dynamics inside the mesopores comes into play when the ratio of mesopores to micropores surpasses 3:1 in pore volume (STC-8). Moreover, after activation, the peak at the cell voltage of $\sim 0.6 \mathrm{~V}$ disappears in the CV curves of Ac-STC-16 (Figure 4a), which indicates that the presence or absence of such a peak is related to the size distribution of the micropores.

Similar to STCs, the nitrogen-doped NDSTCs exhibit an ideal double-layer capacitance behavior, as indicated by the regular shape of the CV curves (Figure 5a and Figure S7f-j), the symmetrical triangle shapes with some small voltage drops of GCPL curves (Figure S7ko), as well as the nearly vertical Nyquist plots in the low-frequency region (Figure S7p-t, except NDSTC-1 because of its partially inaccessible micropores). Compared with the CV curves of the STCs (Figure 3a and Figure S7a-e), the peaks at the cell voltage of $\sim 0.6 \mathrm{~V}$ 
disappear in those of the NDSTCs, resulting in a slight decrease $(\sim 5 \%)$ of capacitance at the low specific current of $0.2 \mathrm{~A} \mathrm{~g}^{-1}$ when all the surface is accessible, except in case of NDSTC1 (Figure 5b-c). The absence of the peak at $\sim 0.6 \mathrm{~V}$ might contribute to the lower capacitance, and at least in the present case nitrogen doping does not improve the capacitance at all. In order to rule out the presence of redox peaks which are invisible in two-electrode configuration, the CV curve of NDSTC-16 was also measured by a three-electrode configuration (Figure S9). The CV curve shows a nearly rectangular shape without redox peaks, in good accordance with the two-electrode configuration. Therefore, it is deduced that our results tested by two-electrode configurations are representative for the electrochemical properties under real potential control. Moreover, it also proves that the nitrogen/oxygen containing functional groups and the trace amounts of $\mathrm{Zn}$ induce no redox peaks in the $\mathrm{CV}$ curve. To further eliminate the possibility of $\mathrm{Zn}$ causing redox peaks in the $\mathrm{CV}$ curves, EDLCs in aqueous electrolytes were tested. All the CV curves of STC-1, NDSTC-1, STC-16, and NDSTC-16 (Figure S10) in the $1 \mathrm{M} \mathrm{Na}_{2} \mathrm{SO}_{4}$ aqueous electrolyte show perfect rectangular shapes without the presence of any redox peaks within the investigated potential range.

Opposite to pure capacitance, a remarkable influence of nitrogen doping on the EDLC performance can be attributed to the improvement of wettability due to specific interactions between IL ions and doped sites in the carbon pore walls, which substantially promotes the ion dynamics inside micropores. This gives the explanation of the $16 \%$ capacitance enhancement of STC-1 after nitrogen doping (Figure 5c). As discussed above, the capacitance of STC-1 at the quasi-equilibrium state is mainly hindered by the poor accessibility of its micropores thus leading to the higher capacitance of NDSTC-1 (Figure 5c) and Ac-STC-1 (Figure S11). The better EDLC performance of NDSTC-1 also indicates the success of doping nitrogen in micropores, consistent with the elemental analysis (Table 1). On the contrary, the capacitance of other STCs is not limited by the ion dynamics inside micropores in the quasi-equilibrium state and thus little influence of the specific capacitance 

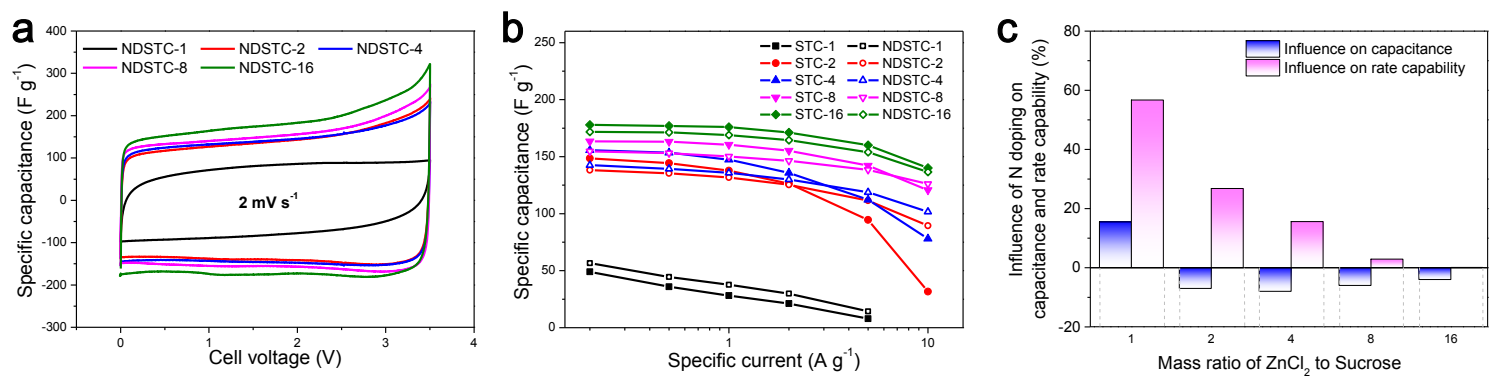

Figure 5. EDLC performance of NDSTCs tested in $\mathrm{EMImBF}_{4}$ ionic liquid using a twoelectrode configuration: (a) Cyclic voltammograms at $2 \mathrm{mV} \mathrm{s}^{-1}$, (b) capacitance retention with specific current increase, and (c) influence of nitrogen doping on specific capacitance at $0.2 \mathrm{~A}$ $\mathrm{g}^{-1}$ and capacitance retention at $5 \mathrm{~A} \mathrm{~g}^{-1}$ compared with the corresponding STCs. 
by the nitrogen doping is observed (Figure 5c). This mechanism also explains the decreasing influence of nitrogen doping on the rate capabilities with the increase of the $\mathrm{ZnCl}_{2}$ (Figure 5c).

It is deduced that the EDLC performance comparison between different carbon electrodes is unaffected by the electric conductivity and is exclusively dependent on the ion dynamics inside the carbon pores. Because the electric conductivities of all these STC and NDSTC electrodes approximate to each other as indicated by the intercepts along the real axis $(\sim 5 \Omega)$ in the EIS measurement (Figure S6b and Figure S7p-t), which is ascribed to their similar degree of aromatization as already reflected by the Raman spectroscopy (Figure S4 and Table S1).

With the highest capacitance and best rate capability among all the samples, STC-16 was selected as the model material to test stability and calculate its specific energy/power. The stability was measured by the voltage floating test, a more demanding benchmarking to survey the electrochemical performance stability compared to voltage cycling. ${ }^{[25]}$ The cell voltage was held at $3.5 \mathrm{~V}$ for $100 \mathrm{~h}$ and interrupted by cycling at $1 \mathrm{Ag} \mathrm{g}^{-1}$ after every $10 \mathrm{~h}$. This approach provides a more strict condition than voltage cycling, which means an EDLC cell is more likely to undergo failure during floating test. A high electrochemical stability of STC-16 was revealed by the desirable capacitance retention of more than $92 \%$ (significantly above the industry-relevant level of $80 \%^{[25]}$ ) and the almost unchanged GCPL curves (Figure 6a). In addition, after cycling at a high charging/discharging rate at $10 \mathrm{~A} \mathrm{~g}^{-1}$ for 10,000 cycles, capacitance retention over $90 \%$ is obtained, indicating its admirable cycling stability at such a high rate. The Ragone plot (Figure 6b) of EDLCs using STC-16 as electrodes with a mass loading of $4.8 \mathrm{mg} \mathrm{cm}^{-2}$ shows that the symmetric cells provide the maximum specific energy of $76 \mathrm{Wh} \mathrm{kg}^{-1}\left(32 \mathrm{Wh} \mathrm{L}^{-1}\right)$ at a specific power of $0.2 \mathrm{~kW} \mathrm{~kg}^{-1}(0.09$ $\left.\mathrm{kW} \mathrm{L}{ }^{-1}\right)$, with a slight drop to $72 \mathrm{Wh} \mathrm{kg}^{-1}\left(31 \mathrm{Wh} \mathrm{L}^{-1}\right)$ at $1 \mathrm{~kW} \mathrm{~kg}^{-1}\left(0.43 \mathrm{~kW} \mathrm{~L}^{-1}\right)$, and still maintains $39 \mathrm{Wh} \mathrm{kg}^{-1}\left(17 \mathrm{Wh} \mathrm{L}^{-1}\right)$ at a specific power of $9 \mathrm{~kW} \mathrm{~kg}^{-1}\left(3.9 \mathrm{~kW} \mathrm{~L}^{-1}\right)$. The specific 
energy is indeed one order of magnitude higher than that of commercial EDLCs, without losing the excellent power capability. This outstanding property is attributed to the optimized pore architecture with all micro- and mesopores, plus the access of a new mode of charge storage, in the volume of the IL rather than only surface double layer compression as it will be discussed below. Furthermore, the correlation of micropores and mesopores to the EDLC performance is also revealed in Ragone plots (Figure 6b). The purely microporous STC-1 shows the worst performance due to the poor accessibility of the micropores to electrolyte ions. With the introduction of mesopores and the increase of their volume, STC-2 and STC-8 exhibit an apparent trend of improvement in both specific power and energy. On the other hand, the performance especially the power density of the STC-8, STC-16, and NDSTC-16 is similar to each other, indicating the further argument of mesopore content or doping with nitrogen have little influence when the ratio of mesopores to micropores surpasses a threshold value of $3: 1$ in volume.

\section{Discussion}

Base on the above results, we propose a model (Scheme 2) to explain how the pore structure and surface chemistry affect the ion migration as well as the structural rearrangements in the bulk electrolyte, thus contributing to the EDLC performance. The inferior EDLC performance of purely microporous carbon (STC-1) even during the quasi-equilibrium charging/discharging process at $0.2 \mathrm{~A} \mathrm{~g}^{-1}$ is ascribed to the poor micropore accessibility caused by the high mobility resistance inside micropores, which can be relieved by introducing mesopores and improving wettability/enlarging micropores, respectively (Scheme 2, first line). Therefore, STC-2 and STC-4 with more mesopores (Figure 3a-c), NDSTC-1 with improved wettability (Figure S7f and k), as well as Ac-STC-1 with larger micropores (Figure S11) exhibit a noticeable enhancement in both specific capacitance and rate capabilities. In comparison, the micropores in the hierarchical micro-mesoporous carbon 

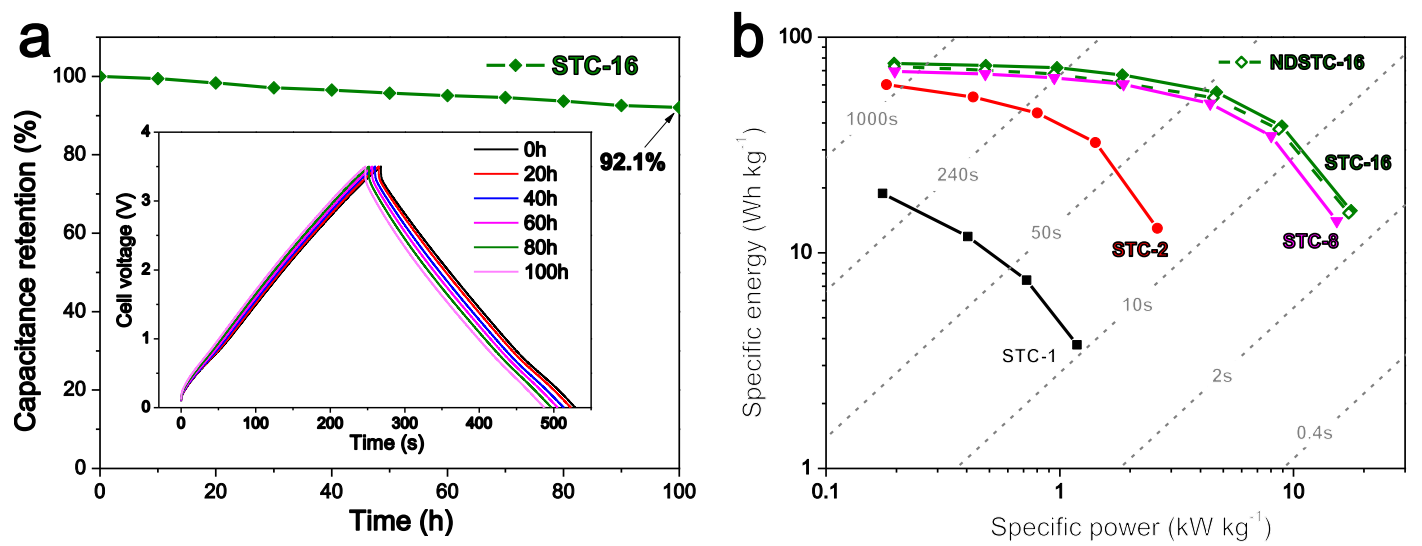

Figure 6. (a) Floating stability of STC-16 at a specific current of $1 \mathrm{~A} \mathrm{~g}^{-1}$ for $100 \mathrm{~h}$ floating at 3.5 V. The inset shows the charge/discharge profiles every $20 \mathrm{~h}$. (b) Ragone plots of EDLCs using STC-1, STC-2, STC-8, STC-16 and NDSTC-16 as electrodes.

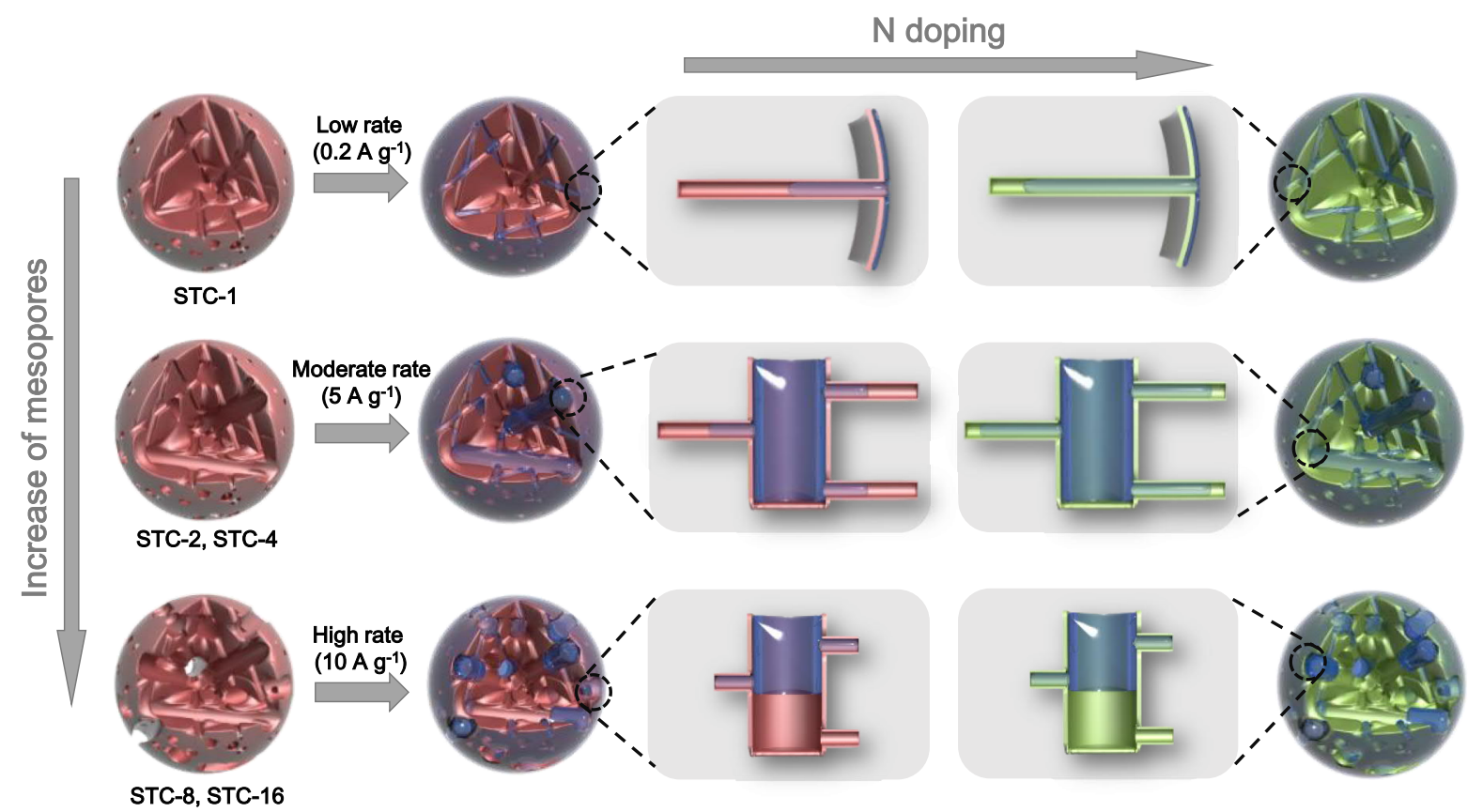

Scheme 2. Influence of different pore structures and nitrogen doping on the ion migration process at different charging/discharging rates. 
(STC-2 and STC-4) are fully accessible at the low charging/discharging rate, as revealed by their remarkably increased capacitance when compared to STC-1. But at the moderate charging/discharging rate of $5 \mathrm{~A} \mathrm{~g}^{-1}$, the ion migration again becomes the limiting factor, leading to the drastic drop of capacitance (Figure 3b). Thus, larger amount of mesopores (STC-8 and STC-16) and nitrogen doping (NDSTC-2 and NDSTC-4) are needed here (Scheme 2, second line), resulting in the more accessible surface areas and higher capacitance at this rate. Nevertheless, at the ultrahigh charging/discharging rate of $10 \mathrm{~A} \mathrm{~g}^{-1}$ even STC-8 and STC-16 with the highest amount of mesopores exhibit a notable capacitance drop (Figure 3b). The rate limiting factor switches from ion dynamics inside micropores to ion transport inside mesopores, as the depth of micropores is already short enough for ions to enter practically instantaneously (Scheme 2, third line). The general limitation for ion transport in presence of large mesopore ratio seems to relate to the viscosity of the electrolyte. As a consequence, it makes no difference to further increase the volume of the mesopores (STC-16 and Ac-STC-16), or dope with nitrogen to reduce the ion migration resistance inside the micropores (NDSTC-8 and NDSTC-16).

The origin of the peaks in the CVs of the STCs at a cell voltage of $\sim 0.6 \mathrm{~V}$ remains an open question. Interestingly, the micropore sizes in the materials are close to the size of EMIm ${ }^{+}$ (Scheme 3). The fact that such peaks are also visible in purely microporous STC-1 and that their area increases at higher micropore accessibility indicates that the process that is responsible for their appearance is taking place in the micropores rather than in mesopores. On an enthalpy scale, $0.6 \mathrm{~V}$ would translate to $\sim 60 \mathrm{~kJ} \mathrm{~mol}^{-1}$ which is in the range of strong inter-molecular forces such as hydrogen-bonds or Coulomb dissociation. To our opinion, the origin of the peak is likely due to rearrangements of the ions in the micropores or changes in their internal conformation due to reversible formation and release of inter-molecular or intramolecular forces on the energy scale of strong secondary valences (Scheme 3, first line). This process is fully reversible as revealed by the small peak at the same cell voltage of $\sim 0.6 \mathrm{~V}$ 
during discharging in the CV curves (Figure 3a) and the superior stability (Figure 6a). It can thus be concluded that the actual energy storage mechanism in EDLCs with IL electrolytes is based on contributions of double-layer formation plus energy stored by solvation of single IL units and their placement in appropriately sized micropores, which is also in agreement with some literature. ${ }^{[18 \mathrm{a}, 18 \mathrm{~b}]}$ In the case of the NDSTCs with the same micropore size, the ion-pore wall interactions are likely to be stronger and more diverse caused by the (weakly) nitrogendoped polar surface. ${ }^{[7 b]}$ In this case, the relative energy change throughout pore integration is lower and also distributed, and thus a narrow peak due to molecular rearrangements of IL ions in the micropores is not observed (Scheme 3, second line). However, also in this case the specific binding between carbon and IL ions will contribute to energy storage in the electrode in addition to double-layer formation. Considering that, it makes sense that hierarchical micro- and mesoporous STCs and NDSTCs show comparable capacities under quasiequilibrium conditions although their energy storage mechanisms suggested by the CVs seem to be different at first glance. The fact that changes in the molecular and intermolecular structure of the ILs also contribute to the energy storage in the mesopores is, as smeared over the whole voltage range, maybe less obvious, but even more relevant for the practical use of such supercapacitors.

In addition to the (later vanishing) peaks at $\sim 0.6 \mathrm{~V}$ during charging and discharging, the overall charge density is simply too high for ordinary double layers, plus there is another pronounced peak at very high voltage in the CVs of all mesopore-containing materials discussed here not related to material degradation (Figure 3). This voltage, when put on a single molecule, would translate to a much higher enthalpy of above $300 \mathrm{~kJ} \mathrm{~mol}^{-1}$ which is ten times the value of a typical heat of vaporization, i.e. the resolution of all intermolecular forces. This makes it clear that such transitions must be related to collective properties and in structural transitions in the bulk of the IL rather than in the electrolyte-carbon interface. 


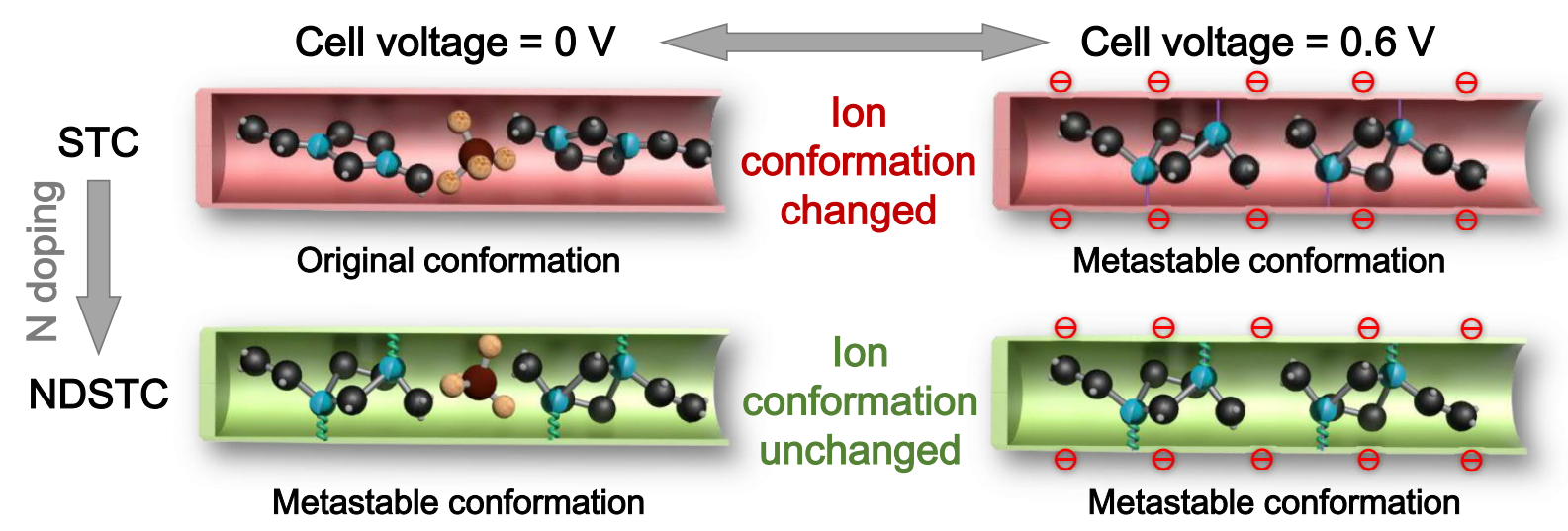

Scheme 3. Proposed mechanism of the ion conformation change inside micropores, causing the emergence of the small peaks in the CV curves of STCs and their disappearance in case of NDSTCs and Ac-STCs. 
A cell voltage of $3.5 \mathrm{~V}$ and specific capacitance up to $178 \mathrm{~F} \mathrm{~g}^{-1}$ relates to a stored charge of $623 \mathrm{C} \mathrm{g}^{-1}$. This is remarkable considering the huge size of the ions of IL electrolytes. It is illustrative to calculate the surface area occupied by one charge. Taking STC-16 as an example, the large $\mathrm{EMIm}^{+}$ions $(0.76 \times 0.43 \mathrm{~nm})$ must be attributed to a calculated surface area per ion of $0.6 \mathrm{~nm}^{2}$ which is already very close to the molecular dimensions of these ions, implying a close to dense layer of cations on the carbon surface. In STC-2 with a lower mesopore ratio, $\mathrm{EMIm}^{+}$ions are formally even attributed to a calculated area of only $0.44 \mathrm{~nm}^{2}$. Such a high double-layer compression appears to be unrealistic for large IL ions in absence of solvent molecules. In contrast to aqueous and organic electrolytes, the density of cations and anions in ionic liquids should stay close to the bulk value, i.e. the energy is essentially not stored in ion compression, but rather in ion rearrangement towards Coulombic higher voltage situations, which obviously can propagate about nanometers into the bulk.

We do not doubt contribution of double-layer formation on carbon surfaces to the energy storage in IL electrolytes in general but from our results we conclude the absence of doublelayer compression as present in aqueous and organic electrolytes, as it has already been shown. ${ }^{[1 \mathrm{~d}, 17]}$ Instead, there seems to be a strong contribution of structural changes in the bulk phase of the ILs to the energy storage mechanism in EDLCs.

This indeed would request a paradigm change of energy storage, as then the majority of energy is not stored in micropores (and cannot be related to a high specific surface area), but rather in appropriately designed mesopores which enable high energy Coulombic rearrangements of the IL in the bulk. In these mesopores, the structural changes of IL ions can be rather assigned to disorder-order transitions in the bulk of the electrolyte than interactions between single ions and the surface, as it is likely the case in micropores. It has been shown that the present family of bulk IL are actually liquid crystals and the peak could, for instance, originate from the formation and disappearance of differently organization schemes of the bulk IL in mesopores. ${ }^{[26]}$ According to this view, such contributions cannot be observed in 
microporous STC-1 and NDSTC-1, and their intensity increases with larger mesopore contribution in the carbon materials. The process is fully reversible as indicated by the high stability of the devices and the nearly perfect symmetrical charge-discharge curves at low current densities.

Based on the present data, we regard it as experimentally proven that the energy storage mechanism in such ionic liquid supercapacitors significantly deviates from the traditional theory of double-layer compression as present in aqueous and organic electrolytes and that there is a notable contribution of ion-ion rearrangements in the bulk of the ionic liquids to the energy storage mechanism in IL-based EDLCs.

\section{Conclusion}

A series of hierarchical porous carbon with different amounts of well-defined micropores and mesopores, large specific surface areas up to $2324 \mathrm{~m}^{2} \mathrm{~g}^{-1}$ and high pore volumes exceeding $1.65 \mathrm{~cm}^{3} \mathrm{~g}^{-1}$ was synthesized. The pore structure, electronic properties, and surface chemistry of the electrode materials is adjusted elaborately by tailoring the ratio of salt templates to carbon precursor and doping with nitrogen, so that the influence of these parameters to EDLC performance could be thoroughly investigated in a homogeneous set of samples. EDLCs based on STC electrodes achieve high specific capacitance of $178 \mathrm{Fg}^{-1}$ at $0.2 \mathrm{~A} \mathrm{~g}^{-1}$ as well as excellent rate capability $\left(140 \mathrm{~F} \mathrm{~g}^{-1}\right.$ at $\left.10 \mathrm{~A} \mathrm{~g}^{-1}\right)$ combined with an admirable stability, using the ionic liquid model electrolyte of $\mathrm{EMImBF}_{4}$ with the voltage window of 0-3.5 V.

In terms of ion dynamics, a model is proposed, where the increasing amount of mesopores and nitrogen doping can promote the EDLC performance only when the ion dynamics inside micropores areas are the rate limiting factor. Ion dynamics inside mesopores commence to limit the EDLC performance at ultrahigh rates when the ratio of mesopores to micropores surpasses 3:1 in volume. Above this ratio, either increase of mesopores or nitrogen doping will have little influence. 
Moreover, a peak in the CV curves of the STCs was observed, and ascribed to an ion conformation change inside micropores during the charging/discharging process. Such additional energy storage due to ion conformation change (i.e., the formation of a metastable phase) is also taking place in NDSTCs but is not observed as peak in the CVs due to the stronger interactions of the IL molecules with the carbon surface leading to non-equilibrium conformation of IL ions before charging. A comparable mechanism in mesopores can be deduced from the formally too-high Coulombic loading and an onset of another peak in the CVs apparent at higher voltages, both related to bulk structural changes simply by the amount of energy involved.

The precise insights into structure-performance relationships enabled by our series of samples let us conclude that there is a significant contribution to energy storage due to molecular rearrangements and phase changes into the bulk of the electrolytes in addition to charge storage in the electric double layer. Decoupling their respective contributions in carbon materials with different pore sizes and surface chemistries holds much promise for further targeted improvement of IL-based energy storage devices.

Based on our findings, the introduction of specific adsorption sites to strengthen the electrodeelectrolyte interaction and thus to stabilize high energy electrolyte modifications is a more promising way to further increase the energy density of EDLCs than striving for ever-higher micropore volumes and SSAs. Finally, the uncovered mechanism offers new possibilities in modifying and optimizing the energy storage mechanisms in IL-based supercapcitors by controlled adjustments of the electrolyte composition (i.e., presence of traces of inorganic salts, traces of solvents, or variations of the ionic liquid ions themselves).

\section{Experimental Section}

Synthesis of STCs: $\mathrm{ZnCl}_{2}$ was employed as the salt template for the synthesis of STCs. In a typical process, $30 \mathrm{~mL}$ aqueous solution of $5 \mathrm{~g}$ sucrose, a certain amount of $\mathrm{ZnCl}_{2}$ and $0.55 \mathrm{~g}$ 
concentrated sulfuric acid was dried at $100{ }^{\circ} \mathrm{C}$ for $6 \mathrm{~h}$, before being heated to $160^{\circ} \mathrm{C}$ and kept for $6 \mathrm{~h}$. The amount of $\mathrm{ZnCl}_{2}$ is determined by the mass ratio of the salt template to sucrose regarding different STCs (Table 1). The mixture was then transferred to a horizontal tubular furnace for carbonization at $900{ }^{\circ} \mathrm{C}$ for $2 \mathrm{~h}$ under $\mathrm{N}_{2}$ flow with heating rate of $1{ }^{\circ} \mathrm{C} \min ^{-1}$. Synthesis of NDSTCs: The STC was dispersed in the aqueous solution of cyanamide $\left(1 \mathrm{~g} \mathrm{~mL}^{-}\right.$ ${ }^{1}$ ) with the mass ratio of STC to cyanamide of $1: 2$, followed by drying at $60{ }^{\circ} \mathrm{C}$ for $12 \mathrm{~h}$ under air. After calcination of the mixture at $800{ }^{\circ} \mathrm{C}$ for $4 \mathrm{~h}$ under $\mathrm{N}_{2}$ flow at with a heating rate of $1^{\circ} \mathrm{C} \mathrm{min}^{-1}$, the corresponding NDSTC was obtained.

Synthesis of Ac-STCs: Ac-STCs were synthesized by $\mathrm{CO}_{2}$ activation of the STCs. Typically, a certain amount of the as-prepared STC was put in a horizontal tubular furnace for thermal treatment at $800{ }^{\circ} \mathrm{C}$ for $0.5 \mathrm{~h}$ under $\mathrm{CO}_{2}$ flow with a heating rate of $10{ }^{\circ} \mathrm{C} \mathrm{min}^{-1}$ to get the corresponding Ac-STC.

Material characterization: $\mathrm{N}_{2}$ physisorption experiments were carried out at $-196{ }^{\circ} \mathrm{C}$ on a Quadrasorb apparatus (40-60 mg sample) from Quantachrome Instruments. Prior to all measurements, the samples were outgassed at $150{ }^{\circ} \mathrm{C}$ for $20 \mathrm{~h}$ under vacuum. SSAs were calculated using the multi-point Brunauer-Emmett-Teller $(B E T)$ model $\left(\mathrm{p} / \mathrm{p}_{0}=0.05-0.2\right)$. Total pore volumes $\left(\mathrm{V}_{\mathrm{t}}\right)$ were determined at $\mathrm{p} / \mathrm{p}_{0}=0.95$. Pore size distributions were calculated using the quenched-solid density functional theory (QSDFT) method (adsorption branch kernel) for $\mathrm{N}_{2}$ adsorbed on carbon with a slit/cylindrical pore shape at $-196{ }^{\circ} \mathrm{C}$ from the data obtained by the low-pressure measurements. $\mathrm{CO}_{2}$ physisorption experiments were carried out at $0{ }^{\circ} \mathrm{C}$ on the same instrument to investigate the pores with the diameter smaller than $1.5 \mathrm{~nm}$. Corresponding PSDs were calculated by the non-local density functional theory (NLDFT) method for $\mathrm{CO}_{2}$ adsorbed on carbon at $0{ }^{\circ} \mathrm{C}$.

X-ray photoelectron spectroscopy (XPS) was performed on a spectrometer equipped with $\mathrm{Al}_{\mathrm{K} \alpha}$ anode $(\mathrm{hv}=1486.6 \mathrm{eV})$. Elemental compositions were determined using survey scans over a range of $1350-0 \mathrm{eV}$ with a step size of $1.0 \mathrm{eV}$ and a pass energy of $200 \mathrm{eV}$. 
Quantitative characterization of the presence of various nitrogen and oxygen sites were accomplished with high-resolution XPS, scanning over a range of $410-392 \mathrm{eV}$ and 544$524 \mathrm{eV}$, respectively, with a step size of $0.1 \mathrm{eV}$ and pass energy of $50 \mathrm{eV}$.

Further structural characterization was carried out by transmission electron microscopy (TEM, EM 912 Omega/Carl-Zeiss Oberkochen) operating at $120 \mathrm{kV}$. To prepare the TEM samples, carbon particles were dispersed in ethanol by sonication for $10 \mathrm{~min}$, followed by dropping several droplets on a carbon-coated copper TEM grid and drying at room temperature. Raman spectra were recorded using a Witec Raman Microscope operating at an excitation wavelength of $532 \mathrm{~nm}$ with a power of $4.0 \mathrm{~mW}$. After the background noise was subtracted from the obtained Raman spectra, the $\mathrm{D}, \mathrm{D}^{2}, \mathrm{~A}$, and $\mathrm{G}$ bands were fitted with a Lorentz function. Thermogravimetric analysis (TGA) was conducted with a Netzsch TG 209 F1 device under constant artificial air flow in platinum pans at a heating rate of $10{ }^{\circ} \mathrm{C} \mathrm{min}^{-1}$ to $1000^{\circ} \mathrm{C}$.

Fabrication of EDLCs and electrochemical measurements: To prepare free-standing electrodes for EDLCs, carbon materials and polytetrafluoroethylene (PTFE, $60 \mathrm{wt} \%$ solution in $\mathrm{H}_{2} \mathrm{O}$ from Sigma Aldrich) were mixed with a mass ratio of $\sim 9: 1$ in ethanol. The solution was then transferred to a glass plate and mixed with razor blades until it changed to a rubberlike consistency. Then it was placed into aluminum foil and rolled to uniformly thin sheets (thicknesses $120 \pm 10 \mu \mathrm{m}$ ) using a commercial roll mill, followed by punching into freestanding electrode disks of $10 \mathrm{~mm}$ in diameter. The areal loading of an electrode was above $3 \mathrm{mg} \mathrm{cm}^{-2}$. The electrodes were dried at $120^{\circ} \mathrm{C}$ for $12 \mathrm{~h}$ under vacuum. EDLCs were tested with a symmetrical two-electrode configuration employing either the ionic liquid 1-ethyl-3methylimidazolium tetrafluoroborate $\left(\mathrm{EMImBF}_{4}, \geq 99.0 \%\right.$, Sigma Aldrich) or $1 \mathrm{M} \mathrm{Na}_{2} \mathrm{SO}_{4}$ in $\mathrm{H}_{2} \mathrm{O}$ as the electrolyte. A Swagelok cell was assembled using a pair of circular electrodes sandwiching a separator (Dreamweaver Silver, $13 \mathrm{~mm}$ in diameter), with $20 \mu \mathrm{L}$ electrolyte and two platinum foils as current collectors. For the test in three-electrode configuration, 
another piece of PVDF-bound activated carbon was introduced from the side to a custommade cell ${ }^{[27]}$ and served as quasi-reference electrode (reference potential: $190 \pm 10 \mathrm{mV}$ versus ferrocene). ${ }^{[27]}$ The counter electrode was made of PTFE-bound NDSTC-16 and largely oversized in charge capacity. All other test conditions are chosen similar to the two-electrode configuration. The EDLCs with IL as electrolytes were assembled in an argon filled glove box $\left(\mathrm{H}_{2} \mathrm{O}<0.1 \mathrm{ppm}, \mathrm{O}_{2}<0.1 \mathrm{ppm}\right)$. A Biologic MPG-2 galvanostat/potentiostat was used for electrochemical characterization. All measurements were performed at room temperature. The electrochemical impedance spectroscopy was performed at open circuit potential with a sinusoidal signal over a frequency range from $20 \mathrm{kHz}$ to $10^{-2} \mathrm{~Hz}$ at an amplitude of $10 \mathrm{mV}$.

Cyclic voltammetry $(\mathrm{CV})$ tests were performed at scan rates of $2-100 \mathrm{mV} \mathrm{s}^{-1}$. The carbon differential gravimetric capacitance for $\mathrm{CV}$ plots, $C_{d}\left(\mathrm{~F} \mathrm{~g}^{-1}\right)$ was calculated according to the following equations:

$$
C_{d}=\frac{2 I}{m \gamma}
$$

where $I$ is the current (A), $\gamma$ is the scan rate $\left(\mathrm{V} \mathrm{s}^{-1}\right)$, and $m$ is the active mass in a single carbon electrode $(\mathrm{g})$.

Galvanostatic charge/discharge with potential limitation (GCPL) was applied at specific currents between 0.2 and $10 \mathrm{Ag}^{-1}$ in a voltage range from 0 to $+3.5 \mathrm{~V}$. The carbon gravimetric capacitance, $C\left(\mathrm{~F} \mathrm{~g}^{-1}\right)$, was calculated according to the following equation:

$$
C=\frac{2 Q_{\text {dis }}}{\left(V-V_{\text {drop }}\right) m}
$$

where $Q_{\text {dis }}(\mathrm{C})$ is the charge of the discharging cycle, $V(\mathrm{~V})$ is the discharging potential change, $V_{\text {drop }}(\mathrm{V})$ is the voltage drop at the beginning of the discharge, and $m(\mathrm{~g})$ is the active mass in a single carbon electrode.

The specific energy, $E\left(\mathrm{Wh} \mathrm{kg}^{-1}\right)$ and specific power, $P\left(\mathrm{~W} \mathrm{~kg}^{-1}\right)$ of EDLCs were estimated via:

$$
E=\frac{1}{8 \times 3.6} C\left(V-V_{d r o p}\right)^{2}
$$




$$
P=\frac{E \times 3600}{\Delta t}
$$

where $V(\mathrm{~V})$ is the discharging potential change, $V_{\text {drop }}(\mathrm{V})$ is the voltage drop at the beginning of the discharge, $\Delta t(\mathrm{~s})$ is the discharging time.

For long-term stability tests, the voltage of the cell was kept at $3.5 \mathrm{~V}$ for $100 \mathrm{~h}$, and the specific capacity was measured every $10 \mathrm{~h}$ by galvanostatic cycling at $1 \mathrm{~A} \mathrm{~g}^{-1}$. In addition, the cycling stability test is accomplished by charging and discharging the cell at a specific current of $10 \mathrm{~A} \mathrm{~g}^{-1}$ for 10,000 cycles.

\section{Supporting Information}

Supporting Information is available from the Wiley Online Library or from the author.

\section{Acknowledgements}

R.Y. acknowledges financial support from China Scholarship Council. All authors thank Dr. Johannes Schmidt (Technical University Berlin, Group of Functional Materials lead by Prof. Dr. Arne Thomas) for providing the XPS data.

Received: ((will be filled in by the editorial staff)) Revised: ((will be filled in by the editorial staff)) Published online: ((will be filled in by the editorial staff))

\section{References}

[1] a) P. Simon, Y. Gogotsi, Acc. Chem. Res. 2012, 46, 1094; b) G. Wang, L. Zhang, J. Zhang, Chem. Soc. Rev. 2012, 41, 797; c) J. R. Miller, P. Simon, Science 2008, 321, 651; d) M. Salanne, B. Rotenberg, K. Naoi, K. Kaneko, P. L. Taberna, C. P. Grey, B. Dunn, P. Simon, Nat. Energy 2016, 1; e) P. Simon, Y. Gogotsi, Nat. Mater. 2008, 7, 845.

[2] a) L. Borchardt, M. Oschatz, S. Kaskel, Mater. Horiz. 2014, 1, 157; b) H. Jiang, P. S. Lee, C. Li, Energ Environ Sci 2013, 6, 41.

[3] a) C. Zhong, Y. D. Deng, W. B. Hu, J. L. Qiao, L. Zhang, J. J. Zhang, Chem. Soc. Rev. 2015, 44, 7484; b) K. L. Van Aken, M. Beidaghi, Y. Gogotsi, Angewandte Chemie International Edition 2015, 54, 4806.

[4] a) M. Davenport, A. Rodriguez, K. J. Shea, Z. S. Siwy, Nano Lett. 2009, 9, 2125; b) C. Pean, C. Merlet, B. Rotenberg, P. A. Madden, P. L. Taberna, B. Daffos, M. Salanne, P. Simon, ACS Nano 2014, 8, 1576; c) N. N. Rajput, J. Monk, R. Singh, F. R. Hung, J. Phys. Chem. C 2012, 116, 5170.

[5] Y. D. He, R. Qiao, J. Vatamanu, O. Borodin, D. Bedrov, J. S. Huang, B. G. Sumpter, J Phys Chem Lett 2016, 7, 36.

[6] a) S. Kondrat, P. Wu, R. Qiao, A. A. Kornyshev, Nat. Mater. 2014, 13, 387; b) S. Kondrat, A. A. Kornyshev, Nanoscale Horiz 2016, 1, 45; c) C. Iacob, J. R. Sangoro, P. 
Papadopoulos, T. Schubert, S. Naumov, R. Valiullin, J. Karger, F. Kremer, Phys. Chem. Chem. Phys. 2010, 12, 13798.

[7] a) R. Singh, J. Monk, F. R. Hung, J. Phys. Chem. C 2011, 115, 16544; b) R. Singh, N. N. Rajput, X. X. He, J. Monk, F. R. Hung, Phys. Chem. Chem. Phys. 2013, 15, 16090; c) J. Monk, R. Singh, F. R. Hung, J. Phys. Chem. C 2011, 115, 3034.

[8] Y. D. He, J. S. Huang, B. G. Sumpter, A. A. Kornyshev, R. Qiao, J Phys Chem Lett 2015, 6, 22.

[9] N. N. Rajput, J. Monk, F. R. Hung, J. Phys. Chem. C 2012, 116, 14504.

[10] a) M. Oschatz, L. Borchardt, K. Pinkert, S. Thieme, M. R. Lohe, C. Hoffmann, M. Benusch, F. M. Wisser, C. Ziegler, L. Giebeler, M. H. Rümmeli, J. Eckert, A. Eychmüller, S. Kaskel, Adv. Energy Mater. 2014, 4, 1300645; b) M. Oschatz, S. Boukhalfa, W. Nickel, J. T. Lee, S. Klosz, L. Borchardt, A. Eychmuller, G. Yushin, S. Kaskel, J. Mater. Chem. A 2014, 2, 5131; c) R. Yan, T. Heil, V. Presser, R. Walczak, M. Antonietti, M. Oschatz, Advanced Sustainable Systems 2017, 1700128; d) M. Oschatz, S. Boukhalfa, W. Nickel, J. P. Hofmann, C. Fischer, G. Yushin, S. Kaskel, Carbon 2017, 113, 283.

[11] A. C. Forse, C. Merlet, P. K. Allan, E. K. Humphreys, J. M. Griffin, M. Aslan, M. Zeiger, V. Presser, Y. Gogotsi, C. P. Grey, Chem. Mater. 2015, 27, 6848.

[12] a) J. H. Hou, C. B. Cao, F. Idrees, X. L. Ma, ACS Nano 2015, 9, 2556; b) J.-K. Ewert, D. Weingarth, C. Denner, M. Friedrich, M. Zeiger, A. Schreiber, N. Jäckel, V. Presser, R. Kempe, J. Mater. Chem. A 2015, 3, 18906.

[13] P. Wu, J. S. Huang, V. Meunier, B. G. Sumpter, R. Qiao, J Phys Chem Lett 2012, 3, 1732.

[14] a) M. D. Levi, N. Levy, S. Sigalov, G. Salitra, D. Aurbach, J. Maier, J. Am. Chem. Soc. 2010, 132, 13220; b) W.-Y. Tsai, P.-L. Taberna, P. Simon, J. Am. Chem. Soc. 2014, 136,8722 .

[15] A. C. Forse, J. M. Griffin, C. Merlet, P. M. Bayley, H. Wang, P. Simon, C. P. Grey, J. Am. Chem. Soc. 2015, 137, 7231.

[16] F. W. Richey, B. Dyatkin, Y. Gogotsi, Y. A. Elabd, J. Am. Chem. Soc. 2013, 135, 12818.

[17] A. C. Forse, C. Merlet, J. M. Griffin, C. P. Grey, J. Am. Chem. Soc. 2016, 138, 5731.

[18] a) D. E. Jiang, J. Z. Wu, J Phys Chem Lett 2013, 4, 1260; b) C. Merlet, B. Rotenberg, P. A. Madden, P. L. Taberna, P. Simon, Y. Gogotsi, M. Salanne, Nat. Mater. 2012, 11, 306; c) S. Kondrat, C. R. Perez, V. Presser, Y. Gogotsi, A. A. Kornyshev, Energ Environ Sci 2012, 5, 6474; d) Y. Shim, H. J. Kim, ACS Nano 2010, 4, 2345; e) C. Largeot, C. Portet, J. Chmiola, P.-L. Taberna, Y. Gogotsi, P. Simon, J. Am. Chem. Soc. 2008, 130, 2730 .

[19] a) N. Fechler, T. P. Fellinger, M. Antonietti, Adv. Mater. 2013, 25, 75; b) N. Fechler, S.-A. Wohlgemuth, P. Jäker, M. Antonietti, J. Mater. Chem. A 2013, 1, 9418.

[20] J. Zhong, J. J. Deng, B. H. Mao, T. Xie, X. H. Sun, Z. G. Mou, C. H. Hong, P. Yang, S. D. Wang, Carbon 2012, 50, 335.

[21] M. Li, W. Li, S. X. Liu, Carbohydr. Res. 2011, 346, 999.

[22] a) M. Oschatz, P. Pré, S. Dörfler, W. Nickel, P. Beaunier, J.-N. Rouzaud, C. Fischer, E. Brunner, S. Kaskel, Carbon 2016, 105, 314; b) S. Osswald, J. Chmiola, Y. Gogotsi, Carbon 2012, 50, 4880.

[23] M. Pawlyta, J.-N. Rouzaud, S. Duber, Carbon 2015, 84, 479.

[24] J. Yang, H. Wu, M. Zhu, W. Ren, Y. Lin, H. Chen, F. Pan, Nano Energy 2017, 33, 453.

[25] D. Weingarth, H. Noh, A. Foelske-Schmitz, A. Wokaun, R. Kötz, Electrochim. Acta 2013, 103, 119.

[26] Y. Zhou, J. H. Schattka, M. Antonietti, Nano Lett. 2004, 4, 477. 
[27] D. Weingarth, M. Zeiger, N. Jackel, M. Aslan, G. Feng, V. Presser, Adv. Energy Mater. 2014, 4, 1400316. 